\title{
Newton's Conception of a Limit as interpreted by Jurin and Robins respectively.
}

\author{
By Professor George A. Gibson. \\ (Read and Received 12th May 19:2.)
}

In his recent book $A$ IIistory of the Conceptions of Limits and Fluxions in Great Britain from Nerton to Woodhouse (Chicago and London: The Open Court Publishing Company, 1919), as well as in a series of articles in the American Mathematical Monthly for 1915 on The IIistory of Zeno's Arguments on Motion, Mr Cajori discusses certain aspects of the conception of a limit, and treats in considerable detail the controversy between Jurin and Robins that arose out of the publication of Berkeley's Analyst. $I$ gave an account of the controversy in a paper that appears in Volume XVII. of our Proceedings, and as Mr Cajori's estimate of the respective merits of the contributions by Jurin and Robins differs greatly from mine, and as the conception of a limit is fundamental in modern mathematics I venture to draw the attention of the Society to the matter.

Mr Cajori's general standpoint may perhaps be fairly represented by the Remarks (History, pp. 146-148) in which he sums up his discussion of Jurin's controversy with Robins and Pemberton

"The debate between Jurin and Robins is the most thorough discussion of the theory of limits carried on in England during the eighteenth century. It constitutes a refinement of previous conceptions.

"Jurin possessed the more general conception of a limit in insisting that there are variables which reach their limits. His interpretation of Newton on this point appears to us more nearly correct than that of Robins; Jurin's geometric illustrations of limit-reaching variable, intended to aid the imagination, though as he admits incapable of exhibiting the process 'all the way,' are nevertheless interesting. The imagination is subject to limitations where the reason is still free to act. 
"Robins, and after him Pemberton, deserve credit in clearly, openly and completely breaking away from infinitely little quantities and from prime and ultimate ratios. Robins's conception of a limit was narrow, but his narrowness had certain pedagogical advantages since it did not involve a mode of advance to the limit which altogether transcended the power of the imagination to follow all the way.

"It is interesting to observe that both Jurin and Robins disavow belief in the possibility of a subdivision of a line into parts so as to reach a point-they assert 'that such subdivision can never be actually finished.'

"Robins discarded the use of Newton's moments in developing the theory of fluxions.

"Towards the end of the long debate with Robins, Jurin begins to disavow infinitely small quantities. He brings out the difference between infinitesimals as variables and infinitesimals as constants. He rejects all quantity ' $f$ ixed, determinate, invariable, indivisible, less than any finite quantity whatsoever,' but he usually admits somewhat hazily a quantity 'variable, divisible, that by a constant diminution is conceived to become less than any finite quantity whatever, and at last to vanish into nothing.' "

In the present paper the phrases "limit-reaching variable," "reaches its limit" and the like frequently occur. To prevent ambiguity I state the sense in which I understand them.

If the function $f(x)$ tends to the limit $l$ when $x$ tends to $a$, and if $f(x)$ has a definite value when $x$ is equal to $a$, then $f(x)$ is said to reach its limit for $x$ tending to $a$, provided that definite value is $l$. If, however, that definite value is not equal to $l$, or if $f(x)$ is not defined for $x=a$, then $f(x)$ does not reach its limit. I assume that a function which is not defined has no value in the sense required for mathematical operations. Thus $\sin x / x$ has a definite limit when $x$ tends to zero, but has no value when $x$ is zero. Again, if we have two concentric spheres, and if the radius of the smaller tends to that of the larger, then the larger sphere is the limit of the smaller and the limit is reached In general terms a variable reaches its limit if, and only if, the limit is at the same time the value of the variable for the corresponding value of the independent variable. 
Now there is certainly a fundamental difference between Jurin and Robins in their conception of a limit, but each maintained that his interpretation of Newton was correct. I shall consider first their respective conceptions.

According to Mr Cajori, Jurin possessed the "more general" conception because he insisted that there are variables which reach their limits, while Robins's conception was "narrow" because it disearded the property of reaching the limit. Mr Cajori states Jurin's position very mildly when he says that "there are variables which reach their limits"; Jurin, I think, considers those cases in which the variable does not reach its limit as some what exceptional. However that may be, it is characteristic of Jurin that he holds that many of Newton's demonstrations are not valid unless the variable actually reaches its limit. (See, for example, Mr Cajori's History, p. 122.)

On what grounds does Mr Cajori characterise Robins's conception as "narrow"? Apparently because in his definition of a limit Robins has the restriction that the variable "can never be made absolutely equal to the limit." It is to be noted, however, that this restrictive clause is not essential. In $\$ 45$ of the Dissertation on the Discourse Robins refers to this point; he there says:- "Whenever the quantities or ratios compared in this lemma [Newton's Lemma I. given below] are capable of an actual equality they must really become so. . . . As in every subject of this lemma all ultimate difference is excluded, the consequences drawn from it are equally just and perspicuous whether the quantities do or do not become actually equal. And this restriction of the sense of this lemma is absolutely necessary to be attended to in this doctrine; because Sir Isaac Newton himself has applied it to quantities and ratios incapable of an actual equality or agreement."

The restrictive clause was manifestly inserted because Berkeley's criticisms depended for their validity on the assumption that Newton's method involved such processes as dividing zero by zero, or making the sum of an "infinite" number of nothings equal to something. The definition was framed to meet such cases and to guard against the supposition that any use was made of the variables in their so-called "prime" or "ultimate" state. As the definition is actually applied by Robins the restriction refers more to the independent than to the dependent variable; the vital 
element is-and he constantly insists on it-that, for example, in finding the limit of $\left\{(x+h)^{n}-x^{n}\right\} / h$ for $h$ tending to zero the value zero is not one that $h$ is to take. It was to meet the argument that Newton put $h$ equal to zero that Robins framed his definition, but he was well aware and, as we have seen, expressly stated that the whole work was quite independent of any assumption regarding the equality of the limit and the value. In fact if the value could be calculated there was no call for using Newton's process and any such case was without interest for the controversy with Berkeley. What Robins saw clearly and emphasised was that in finding the limit of $f(x)$ for $x$ tending to $a$ the value $a$ itself was not to be used, and the conclusions were "equally just and perspicuous" whether the limit was or was not equal to the value. In thus discriminating between limit and value Robins seems to me to anticipate the essential points in the definition of a limit that are so frequently emphasised by modern writer's* if his conception is "narrow" I fear we must conclude that the conception, as expressed in modern treatises, is equally "narrow." It would have made the work of understanding Mr Cajori's criticisms simpler had he given his own definition of a limit; so far as I can see, Robins's conception is identical with current conceptions, even though his precise phraseology is not quite satisfactory.

Whether Jurin's interpretation of Newton was "more nearly correct than that of Robins" is of course a different question. The answer, it seems to me, depends on the weight we attach to the literal meaning of words as contrasted with the meaning assigned to them by a consideration of their setting and of the cautions laid down by Newton in regard to their interpretation. I think there is no doubt that if Newton's language is to be interpreted literally Jurin had much to say for his contentions, but it is worth noting that Jurin himself, when confronted with the illustration used by Newton at the end of the Scholium in Section I., Book I. of the Principia, has to admit that the language of Lemma I. cannot be taken literally in this case (Republic of Letters, November 1735, pp. 374-384 and January 1736, pp. 90-91). Even Jurin therefore was unable to contend that all cases of limits

* See, for example, Hardy, Course of Pure Mathematics (2nd Edition), p. $169, \S 97$. 
could be brought under his interpretation, which he asserted was the only legitimate interpretation of Lemma I., namely, that the variable must be supposed to reach its limit. But Lemma $I$. is fundamental, and it is on the interpretation of that Lemma that the discussion turns.

The Lemmas referred to in the preceding and following pages are those of Section I., Book I. of the Principia, and it is necessary to quote the first two of them.

Lemma I._-"Quantities, and also the ratios of quantities, which in any finite time tend constantly to equality and before the end of that time approach nearer to each other than by any given difference, become ultimately equal."

The proof given is - "If not, let them become ultimately unequal and let their ultimate difference be $D$, therefore they cannot approach nearer to equality than by the given difference $D$; contrary to the hypothesis."

Lemma II.-To determine the area of the figure bounded by the perpendicular lines $a A, A E$ and the curve $a b c d E$ Newton

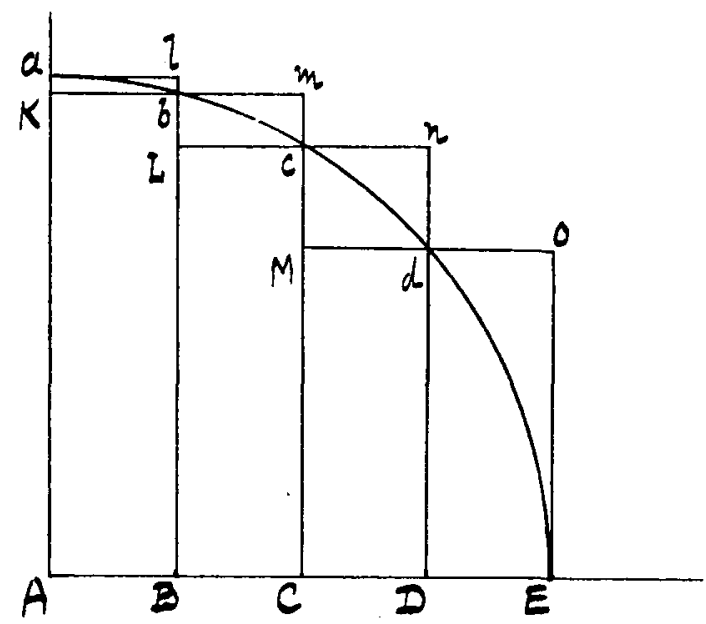

divides the base $A E$ into "any number" of equal parts $A B, b C$, $C D, D E$ and on these parts describes rectangles of which one series forms a circumscribed figure AalbmcndoE and the other forms an inscribed figure $A K b L c_{1} / d D$. He then enunciates the 
proposition:- "Let the width of these rectangles be diminished and their number increased in infinitum; I say that the ultimate ratios which the inscribed figure $A K \ldots d D$, the circumscribed figure $A a \ldots o E$, and the curvilineal figure bave to one another are ratios of equality."

To prove the proposition he notes that the difference between the inscribed and the circumscribed figures is the rectangle $A a l B$. "But this rectangle, since its width is diminished in infinitum, becomes less than any given rectangle. Therefore, by Lemma I., the inscribed and circumscribed figures and, much more, the curvilineal figure which lies between them become ultimately equal."

Now Jurin's contention was that the quantities mentioned in Lemma I. do at last become equal; " these words, when used by a Mathematician, can imply nothing but equality, mathematical, $i e$, absolute, perfect equality." (Rep Let., January 1736, p. 79.) To Robins's question "Does Philalethes here suppose the truth of Sir Isaac Newton's demonstrations to depend on this actual equality of the variable quantity and its limit"? he replies "I do." (Rep. Let., August 1736, p. 128) Jurin is even bold enough to say (Rep. Let., January 1736, p. 89):-“The perimeter of the circumscribed figure, so long as it continues rectilineal, is indeed equal to the sum of the lines $a A, A E$; but when that figure at the instant that the rectangle $A B l a$ vanishes into nothing does thereby come to coincide with the curvilineal figure, as its last form, or its limit which it then arrives at, its perimeter is then no other than the curve itself.

Mr Cajori says " the imagination is subject to limitations where the reason is still free to act." I wonder whether he attributes this transformation of a line of constant length into a curve of a quite different length to the freedom of the reason. Surely the absurdity of the contention that the "last form" of the circumscribed figure is that of the curve is definitely established by the very language in which Jurin expresses himself. Where in reason or imagination do we find any grounds for asserting that the perimeter albmcndo $E$ - whose length is always equal to $a A+A E$ so long as we know anything about it-transforms itself into the curve $a b c d E$ ? If Jurin's interpretation of Newton is more correct than that of Robins I think Newton's reputation as a mathematician must suffer. 
Robins gives a different interpretation of Lemma I. In his view the words "ultimately equal" are defined by the Lemma and its proof; they are not to be interpreted in terms of the separate notions implied in the words "ultimately" and "equal," but the two words are to be taken together as expressing the conception embodied in the conditions which the varying quantities are supposed to satisfy. First there are two varying quantities; next there is a given quantity, which may be any whatever; finally the varying quantities are such that in their variation they become less than the given quantity. When two variable quantities satisfy these conditions they become "ultimately equal" - that is, each tends to a fixed quantity which is the same for both, or, they have the same limit. This interpretation is based on the fact that nowhere in the proof does any varying quantity or magnitude appear in what is called its "last form"; it is only such of their properties as can be deduced from the quantities in their varying form that are ever referred to, and the conclusion depends solely on the fact that, however small be the given difference, the quantities will vary till their difference becomes less than that given difference.

It is to be observed that the phrase "given difference" is the well established language of the ancient geometry and does not include "zero difference."

From the point of view of the theory of limits the theorem of Lemma I. is defective apart from the pbrase "ultimately equal"; all that the Lemma really proves is that if one of the quantities has a limit so has the other, and the limit is the same for both. In the applications made by Newton in these Lemmas the difficulty is got over by the assumption-natural and universal in his daythat, for example in Lemma II., the fixed magnitude is the curve itself. Robins in his exposition prefers a method of stating the case that is more consistent with modern practice. In his first paper, the Discourse on Fluxions, he defines an "ultimate magnitude" as "the limit to which a varying magnitude can approach within any degree of nearness whatever, though it can never be made absolutely equal to it." Thus the curvilineal figure in Lemma II. is "the ultimate magnitude" or "the limit" of the circumscribed (or of the inscribed) figure. With this conception there is now no question of a "last form" of the circumscribed 
figure of Lemma II. The properties dealt with are areas, and since the difference between the areas of the circumscribed and curvilineal figures may be made less than any given area, the curvilineal figure is the "ultimate magnitude" or the limit of the circumscribed; or the circumscribed is "ultimately equal" to the curvilineal figure.

Is this interpretation reasonable? I think it is; not only so, but I do not think that any other interpretation is consistent with Newton's later work. I say "later work" because I believe Robins was correct in maintaining that Newton's early work was based on the use of indivisibles. The strongest grounds for Robins's position seem to me to lie in the demonstrations given by Newton of the Lemmas on areas; nowhere does he ever even suggest that he uses any properties of the varying magnitude except such as are determined by it while still in the varying state. When, for example, he has shown that the difference between the circum. scribed and the curvilineal figures may be made less than any given difference his work is at an end. If he held it to be essential to his conception of an "ultimate magnitude" that it should be a particular case or a "last form" or a value, in any sense of the word, of the varying magnitude it was surely a grave defect in his demonstrations that in no single case does he even try to show the coincidence of the varying rectilineal figure with the curvilineal figure to which it is "ultimately equal."

It is at the same time quite true that Newton's language is often misleading, if the above interpretation is correct. For example, in Lemma III., Cor. 1, he says " the ultimate sum of the vanishing parallelograms coincides completely (omni ex parte) with the curvilineal figure." This sentence, if taken by itself and read without reference to Newton's general caution as to the sense in which his words are to be understood, would be entirely in favour of Jurin's contention. But in the Scholium, at the end of Section I., in which he considers possible objections to the fundamental ideas of the Lemmas he warns the reader against certain misinterpretations. I do not think the Scholium to be a model of lucid exposition, but it contains some very definite statements that are pertinent to the controversy. The last paragraph of the Scholium is specially important. He there says, "it may be contended that if the ultimate ratios of vanishing quantities are given the ultimate 
magnitudes will also be given," and, to put his conceptions in the clearest light, he takes the case of infinitely great quantities. "If two quantities which have a given difference increase in infinitum their ultimate ratio will be given, yet not on that account (ideo) will the ultimate or greatest quantities be given of which that is the ratio." It seems to me that the Scholium loses its cogency unless the considerations adduced in it apply to all the Lemmas; Robins was therefore, in my opinion, justified in his general interpretation of Newton. Newton continued to use frequently the language of indivisibles, and explains in the Scholium that he does so "to facilitate the conception," but he adds an explicit caution on the subject.

The language of indivisibles was then universally in use; is it a matter for surprise that Newton, who had so much weighty matter to expound, did not develope his method of prime and ultimate ratios with the precision of language he might otherwise have tried to attain? He was sure of the fundamental accuracy not only of his results but of his logic; why devote longer time to the presentation of his method in a form that would not be open to the objections that he clearly foresaw? Is it not even now quite common to say, for example, that when $x$ tends to zero " $\sin x / x$ is ultimately equal to unity," or " $\sin x / x$ is equal to unity in the limit"? Those who use such expressions do not mean that unity is the value of $\sin x / x$ when $x$ is zero; it is not $\sin x / x$ that is then equal to anything. What is meant of course is that the limit of $\sin x / x$ is unity, and the word "equal "applies to the word "limit." It may be sometimes convenient to use the phrase " $\sin x / x$ is equal to unity in the limit"; it may fit in better with a smooth running sentence, but those who use it mean simply that the limit is unity.

It is absurd to represent Newton as flawless, and Robins was much readier than Jurin to see and to specify faults in Newton's work, but it is surely going to the other extreme to tie Newton down to "literalism" when there is another method of explanation that is more consistent with his assertions and that relieves him of the charge that at bottom his theory rests on indivisibles. Robins in any case developed a theory of limits that in all essentials agrees with that now in common use, and he always maintained that he learned it from Newton's works. 
How are we to account for Jurin's persistence in maintaining that the circumseribed figure in Lemma II. "does come to coincide with the curvilineal figure as its last form"? I think his contention rests on the illegitimate identification of two quite distinct processes. Consider first the problem of finding the tangent at a given point $P$ on a curve. Take a near point $Q$ on the curve and let the chord $P Q$ be produced to $R$. Now, the length of $P R$ being kept constant, let $P R$ turn about $P$, and suppose the rotation to stop when $Q$ comes into coincidence with $P$. The secant $P R$ in this position is the tangent at $P$. In this way of considering the matter the tangent may be said to be a particular position of the secant or even of the chord, though there is no longer any chord, and it is rather a stretch of language to speak of a chord when it is no longer a chord. Suppose, however, that we try to determine the position of the tangent by calculation; a new element enters because there are rules of calculation which must be observed. When the coordinates of $P$ and $Q$ are known we can calculate the gradient, $\left(y_{2}-y_{1}\right) /\left(x_{2}-x_{1}\right)$ say, of the secant, but we are not at liberty to make $x_{2}$ equal to $x_{1}$ since that would mean a zero divisor. The parallelism with the first mode of considering the tangent breaks down, and it is precisely because it does break down that the method of limits is introduced. I think that Berkeley exposed once for all the futility of "putting $\Delta x$ equal to zero." In the case of the tangent we may "by imagination" follow the course of $Q$ till it actually coincides with $P$ and can see $P R$ move into the position of the tangent, but our "reason" forbids the calculation of the gradient of the tangent by putting $x_{2}$ equal to $x_{1}$, that is by making $Q$ coincide with $P$. The coincidence is impossible in the case of the calculation, and it is quite illegitimate to conclude that because in the method of rotation the secant may be brought inta coincidence with the tangent the same coincidence is possible for the determination of the gradient.

Similar considerations, in my opinion, apply to the illustrations of "limit-reaching variables" given by Jurin. Take for example the illustration represented by the figure on p. 122 of $\mathrm{Mr}$ Cajori's History. $A E=a, C E=x$ and, when $A C$ is an aliquot part of the base $A E$, say $A C=a / n$, then $n-1$ (or $n$ ) is the number of rectangles inscribed in (or circumscribed about) the triangle $B A E$. The ordinate $C d$ is $\frac{1}{2} x$ while the ordinate $C D$ is $a-\frac{1}{2} x$. If we give to $x$ 
the value $a$ each of these ordinates is, no doubt, equal to $\frac{1}{2} a$. But $x=a\left(1-\frac{1}{n}\right)$ and therefore $x$ eannot be equal to $a$ unless $a / n$ is zero; thus with $a$ as the "value" of $x$ we have rectangles with zero width, and the conclusion to be drawn is that the triangle is made up of an "infinite number" of rectangles of zero width. If this is not what Jurin calls "indivisibieism," pure and simple, I am at a loss to know what it is. Berkeley's criticism applies here with full force; $a$ is not a possible value of $x$, it is the limit to which $x$ tends, and the triangle is not the "last form" of either set of rectangles but its area is the limit of the area of either set.

There is another matter to which I wish to refer briefly. Mr Cajori says that "Robins discarded the use of Newton's moments in developing the theory of fluxions." I am not sure what exactly is meant by this statement Robins explains that he had at first considerable difficulty in interpreting Newton's language in a way that did not involve the use of indivisibles-a use that Newton expressly disclaimed-and therefore preferred to develope his exposition without reference to moments. Fuller consideration, however, led him to an interpretation that was independent of indivisibles and was, in his opinion, both sound and consistent with the interpretation he had given of the method of prime and ultimate ratios. (Account of the Discourse, §43.) He had therefore no doubt of the validity of the conception of a moment if understood in his sense. That sense is essentially the same as is involved in the modern conception of a "differential," and I think Robins performed a valuable service in presenting the conception in such a way as to justify confidence in the applications Newton makes of it in the Principia.

In criticising the writings of Newton, Jurin and Robins it would be unreasonable to demand the refinements of modern mathematics. These refinements are based on a long development of conceptions and notations which had their origin to a considerable extent in these writings themselves; without the work of men of the stamp of Newton the development would probably have been much slower. The fundamental conception of a limit is well applied, if not precisely defined, in the Principia and other works of Newton, and is, in my judgment, very clearly expounded by 
Robins. Jurin, it seems to me, stands on a much lower plane; I do not think he ever really advanced beyond the conceptions of the older school who used indivisibles, however strongly he resented such an imputation.

Mr Cajori's History provides a valuable summary of English books on limits and fluxions during the period of which it treats. I venture to differ strongly from some of his estimates, but I hope I have not revived anything of the spirit that unfortunately developed as the controversy between Jurin, Robins and Pemberton displayed itself to the readers of the Republic $f$ Letters and the History of the Works of the Learned. 